\title{
Arterial Stiffness as a Measure of Vascular Dysfunction in Hypertensive Women
}

\author{
Akshatha Nethi ${ }^{1}$ Rajiv Kumar B ${ }^{2}$ Farheen Fatima ${ }^{3}$ Vaishali M. Pauniker ${ }^{3}$ Aswin Kumar $\mathrm{M}^{3}$ \\ Madhuri Taranikanti ${ }^{3}$
}

1Employees' State Insurance Corporation Medical College, Hyderabad, Telangana, India

2Department of General Medicine, Employees' State Insurance

Corporation Medical College, Hyderabad, Telangana, India

${ }^{3}$ Department of Physiology, Employees' State Insurance Corporation

Medical College, Hyderabad, Telangana, India Address for correspondence Akshatha Nethi, MBBS, Employees'
State Insurance Corporation Medical College, Sanathnagar, Hyderabad
500038, Telangana, India (e-mail: akshathanethi@gmail.com).

\begin{abstract}
Hypertension, affecting a third of the world's population is associated with many vascular changes in blood vessels, which include rise of peripheral resistance and arterial stiffness. As there are very few studies done on Indian hypertensive women, this project was taken up to determine the prevalence of arterial stiffness in them. In this study, arterial stiffness is determined by assessing the carotid-femoral pulse wave velocity (C-F PWV). Fifty hypertensive female subjects between the ages 25 and 75 years were selected as subjects after informing the procedure and taking their consent. PWV was obtained from blood (BP) and electrocardiogram (ECG) recordings. They showed that subjects belonged to 3 categories depending on the level of their blood pressure-normal, grade I, and grade II hypertensives. PWV increased progressively with level of hypertension ( $p$ values $=0.0171$ and $<0.0001$, which are significant for hypertensives having current BP equal to grade I and II hypertension, respectively). Vascular remodeling leading to loss of elasticity and

Keywords

- arterial stiffness

- hypertension

- pulse wave velocity

- vascular remodeling impaired relaxation is believed to play a key role in development of arterial stiffness and subsequent rise of PWV in hypertensives. Arterial stiffness causes vascular dysfunction and is known to be an independent risk factor for many cardiovascular diseases like ventricular remodeling, diastolic dysfunction, myocardial infarction, and so forth. Control of Blood Pressure and its sequelae by dietary interventions and regular exercise is advisable.
\end{abstract}

\section{Introduction}

Hypertension is major public health problem globally with nearly $30 \%$ of the population being affected. Chronic hypertension produces anatomical changes to the aorta's tunica media which progressively increases arterial stiffness. This reduces aortic compliance and increases myocardial work and induces changes in the arterial pulse pressure and velocity. Arterial stiffness is linked to hypertension and is a better predictor of future cardiovascular events when compared with sphygmomanometry. ${ }^{1}$

Vascular function is regulated by endothelium and any dysfunction of the blood vessel occurs due to endothelial

published online

March 24, 2020
DOI https://doi.org/ 10.1055/s-0040-1703991. damage or dysfunction, decrease in elastic properties of the arteries, or increase in total peripheral resistance., ${ }^{2,3}$ Vascular dysfunction is the inability of the artery to dilate sufficiently in response to an appropriate endothelial stimulus and characterized by microvascular remodeling, decreased viscoelastic properties of the arteries, and arterioles and abnormal total peripheral resistance (TPR)., ${ }^{3,4}$ Arterial stiffness is of the major contributory factors to multiple cardiovascular diseases like left ventricular hypertrophy (LVH), left ventricular failure (LVF), aneurysms, myocardial ischemia (MI), strokes, and ultimately death.

One of the best measures of arterial stiffness is the pulse wave velocity (PWV). ${ }^{1,5}$ The volume of blood ejected into the 
aorta during the systole generates a pulse wave, circulating through the arterial system with a certain velocity; this is called PWV. It can be measured noninvasively. ${ }^{4}$ It is the difference between two recording sites in the line of pulse travel and the delay between corresponding points on the wave (of pressure or of flow) which are not influenced by wave reflection. The wave front or initial upstroke is the usual point of reference in the two waveforms. Manifestations of arterial stiffness are seen as characteristic alterations in the arterial pressure waves. ${ }^{1,6}$

Carotid-femoral pulse wave velocity (CF-PWV) is recognized as the gold standard for the arterial stiffness assessment. ${ }^{7,8}$

\section{Aim of the Study}

1. To determine arterial stiffness in hypertensive women by means of PWV measurement.

2. To assess any association between pulse wave velocities and degree of rise of blood pressure.

\section{Materials and Methods}

50 women between the ages of 25 and 75 years visiting the outpatient department of General Medicine for treatment of hypertension.

\section{Method}

Clearance for this study was given by the institutional ethics committee of Employees' State Insurance Corporation Medical College, Sanathnagar, Hyderabad, India. Written consent was obtained from the test subjects. A patient information sheet was prepared to note personal details, like name, age, sex, address, occupation, and also about duration of hypertension (HTN); medication or any other intervention being done was also noted.

After taking the details from the subject, tests were conducted on diabetes risk profiler, manufactured by Genesis Medical Systems Pvt. Ltd. (product name: NeuCaRe_Pro; serial no. NCRP/041601; date of manufacture: July 21, 2016; Hyderabad, India). It is an equipment which can do comprehensive testing of multiple physiological functions of the body like cardiovascular testing, autonomic function tests, nerve conduction in peripheral nerves, and pulmonary function tests. For this study, only cardiovascular component was selected which included the following tests:

1. Recording of blood pressure (BP) from both upper and lower limbs was obtained bilaterally.

2. Electrocardiogram (ECG) recording was simultaneously done.

Based on the parameters stated earlier, PWV was obtained.

Timeframe: 1 month.

Study design: Cross-sectional study.

\section{Inclusion and Exclusion Criteria}

Only females, between 25 and 75 years of age, having hypertension.

\section{Exclusion Criteria}

1. Males.

2. Females younger than 25 years and older than 75 years.

3. Having any associated risk factors, for example, smoking.

4. Having any other chronic diseases or undergoing treatment for the same. For example, diabetes mellitus, hypothyroidism, anemia, and so forth.

5. Taking any other drugs which may influence the blood pressure, for example, oral contraceptives.

6. Having undergone any major surgery in the past 6 months.

\section{Statistical Analysis}

Data was entered in Microsoft Excel and graphs were drawn using Microsoft Excel and Microsoft Word.

Student $t$-test (Unpaired $t$-test) was performed using GraphPad Instat Software. A $p$ value of $\leq 0.05$ was considered significant.

Subjects were divided into 3 groups based on the current levels of their blood pressure ${ }^{9,10}$ (- Table 1), as measured on the day of the test in the outpatient department.

1. Subjects having normal values of blood pressure.

2. Subjects exhibiting values of grade I hypertension.

3. Subjects exhibiting values of grade II hypertension.

\section{Classification of Hypertension}

\section{Observation and Results}

For this study, the normal group includes blood pressures $<130 \mathrm{~mm} \mathrm{Hg}$ systolic (SBP) and $<80 \mathrm{~mm} \mathrm{Hg}$ diastolic blood pressure (DBP). ${ }^{9,10}$

Analysis of data was done using GraphPad Instat software. Unpaired student $t$-test was performed.

On comparison between the normotensive group and grade I HTN group, a $p$ value of 0.0171 was obtained, which is significant (- Table 2 and - Fig. 1 ).

When comparing normotensive group with grade II HTN group, $p$ value of $<0.0001$ was obtained, which is extremely significant ( - Table 2 and - Fig. 1).

Table 1 Table showing the classification of hypertension

\begin{tabular}{|l|l|}
\hline Grade & Values of blood pressure $(\mathrm{mm} \mathrm{Hg})$ \\
\hline Normal & $<120$ systolic and $<80$ diastolic \\
\hline Elevated & $120-129$ systolic and $<80$ diastolic \\
\hline Grade I hypertension & $\begin{array}{l}\text { Systolic: } 130-139 \\
\text { Diastolic: } 80-89\end{array}$ \\
\hline Grade II hypertension & $\begin{array}{l}\text { Systolic: }>140 \\
\text { Diastolic: }>90\end{array}$ \\
\hline
\end{tabular}


Table 2 Table showing mean duration of hypertension (HTN), mean values of blood pressure, and carotid-femoral pulse wave velocity (C-F PWV)

\begin{tabular}{|l|l|l|l|}
\hline Grade of HTN $(\boldsymbol{n}=\mathbf{5 0})$ & $\begin{array}{l}\text { Mean duration of } \\
\text { hypertension }\end{array}$ & $\begin{array}{l}\text { Mean values of the blood } \\
\text { pressure }\end{array}$ & $\begin{array}{l}\text { C-F PWV }(\mathrm{cm} / \mathrm{sec}) \\
\text { Mean } \pm \text { SD }\end{array}$ \\
\hline Normal $(\boldsymbol{n}=22)$ & $5.8 \mathrm{y}$ & $124 / 76 \mathrm{~mm} \mathrm{Hg}$ & $1127.61 \pm 64.70$ \\
\hline Grade I HTN $(\boldsymbol{n}=13)$ & $7.4 \mathrm{y}$ & $134 / 89 \mathrm{~mm} \mathrm{Hg}$ & $1421.41 \pm 278.07$ \\
\hline Grade II HTN $(\boldsymbol{n}=15)$ & $5.25 \mathrm{y}$ & $156 / 92 \mathrm{~mm} \mathrm{Hg}$ & $1497.84 \pm 86.26$ \\
\hline
\end{tabular}

\section{COMPARISON OF PULSE WAVE VELOCITY IN} HYPERTENSIVES HAVING VARYING DEGREES OF BLOOD PRESSURE

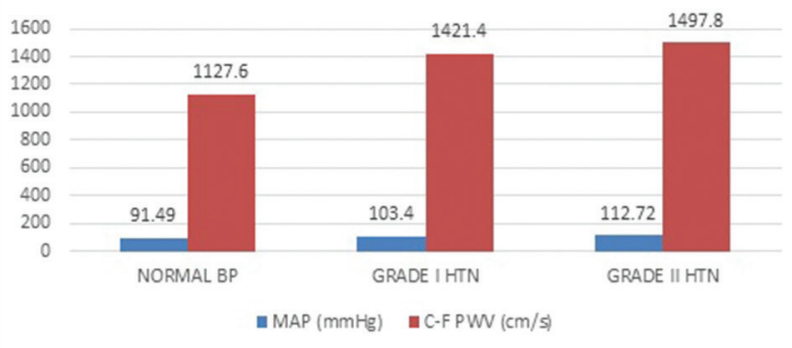

Fig. 1 Graph showing the comparison of pulse wave velocity in hypertensives having varying levels of blood pressure.

\section{Discussion}

Hypertension, one of the leading causes of morbidity and mortality, ${ }_{1}^{11}$ is a major public health problem and a known independent risk factor for cardiovascular diseases. ${ }^{12}$ One of the changes in hypertension is increase in arterial stiffness which causes vascular dysfunction. Pulse wave velocity (PWV), one of the important parameters to determine the presence of arterial stiffness, is used in this study. Only females were selected as subjects as there weren't many studies done on Indian women to determine the association of arterial stiffness with hypertension.

Fifty hypertensive females between the ages of 34 and 68 years were taken as subjects, with mean age being 51.73 years. Personal information and written consent was obtained from all the subjects. After making them rest for 5 to 10 minutes, PWV was estimated on diabetes risk profiler. Diabetes risk profiler is a comprehensive testing equipment which with its associated software can do multiple tests simultaneously, one of which is assessing various vascular parameters. BP can be recorded noninvasively in all four limbs at the same time along with ECG. Based on the above parameters recorded, PWV and arterial stiffness in major vessels of all four limbs is automatically given by the software.

Data was entered into Microsoft Excel sheet and Statistical Analysis was done using student unpaired $t$-test. A $p$ value of $<0.05$ was considered significant.

Even though all subjects were under antihypertensive medications, varied range of blood pressures were recorded in them. Hence, the subjects were divided into 3 groups, based on their current blood pressures ( - Table 1 ):
1. Twenty-two subjects having normal blood pressure, having a mean duration of hypertension of 5.8 years.

2. Thirteen subjects having blood pressures correlating with grade I hypertension, having a mean duration of hypertension of 7.4 years.

3. Fifteen subjects having blood pressures correlating with grade II hypertension, having a mean duration of hypertension of 5.25 years.

Average of mean arterial pressure of each group was calculated. Mean of PWV in each group was also estimated. Presence of any correlation between the two was assessed. It was observed that although C-F PWV increased with presence of hypertension in this study, which is similar to previous studies, ${ }^{13}$ degree of rise in this study was associated with grade of hypertension, more specifically the current values ( - Table 2 and - Fig. 1 ).

Although all subjects were under treatment with standard dosages of antihypertensive medications-both telmisartan, an angiotensin receptor blocker, and amlodipine, a calcium channel blocker, were prescribed concurrently; they showed varying levels of blood pressures when assessed at the time of the test. Hypertensives having higher levels of current blood pressure (corresponding to grade I and grade II hypertension values) had significantly high PWV when compared with hypertensives having normal blood pressure, with a $p$ value of 0.0171 and $<0.0001$, respectively ( - Table 2 and - Fig. $\mathbf{1}$ ).

In this study, the duration of hypertension appears to be playing no significant role in degree of rise of PWV. Subjects with raised blood pressures were advised to get further management from the Department of General Medicine.

Previous studies indicate that vascular changes occurring in hypertension are similar to changes that occur with aging. Aging by itself causes vascular remodeling. In the additional presence of hypertension, these changes get enhanced and occur in an accelerated manner. Microscopic changes include the presence of endothelial cell damage, inflammation, increased proliferation of vascular smooth muscle, increased deposition of extracellular matrix, fibrosis, and calcification, which ultimately lead to increase in stiffness of vascular wall. ${ }^{14}$ All the changes stated earlier lead to mechanical and functional changes like reduced distensibility and elasticity of the vessel wall. Arterial stiffness increases and compliance decreases. Part of this stiffening is also associated with elastin fiber fragmentation in the arterial vessel wall. ${ }^{14}$ 
A group of enzymes from the zinc dependent endopeptidase family are responsible for elastin degradation. These enzymes are known as matrix metalloproteinases (MMP). Specifically, MMP-2 and MMP-9 levels are found to be increased in the vessel wall which is showing age-related changes. ${ }^{15}$ Other than elastin, collagen fibers also undergo structural changes in the vessel wall. Cross linking of collagen fibers by advanced glycation end products (AGEs) is associated with arterial stiffness. Although the popular notion is that AGEs are associated with diabetes mellitus, it has been observed that AGEs can be present in the body at high levels even in nondiabetics, which predisposes to tissue injury and consumes the antioxidant reserves in the body. Studies comparing diabetics taking high AGE meal with low AGE meal, showed increase of endothelial dysfunction with increasing AGE quantity. ${ }^{16}$ In fact, it was found that hypertensives have higher AGE levels than normotensives. ${ }^{17}$ In an animal study, suppression of AGEs by aminoguanidine prevents arterial stiffening without alterations in collagen or elastin content. ${ }^{18}$ An Interventional study was done in hypertensive patients by Kass et al, where they were prescribed ALT-711, a nonenzymatic breaker of collagen cross-links, resulted in a significant reduction in PWV and systolic blood pressure in comparison to placebo without any difference in mean arterial pressure (MAP). ${ }^{19}$

Another possible mechanism contributing to arterial stiffness is impaired production of nitric oxide (NO) by the endothelium, hence relaxation of the vessel wall is impaired. It has been observed that decrease in production of NO occurs with age, but this occurs at a highly accelerated rate in hypertensives. They also have an additional burden of presence of excess oxidative stress. ${ }^{2}$

As evinced by the study done by Stewart et.al, In-vivo arterial stiffness is increased by an acute rise in blood pressure. ${ }^{20}$ Hence, reduction of blood pressure should theoretically reduce arterial stiffness. But in those hypertensives in whom vascular remodeling and structural changes have already set in, reducing the effect of the hypertensive risk factor won't reverse the physical changes, that is, arterial stiffness persists. In fact, sustained hypertension may also accelerate structural changes to the arterial wall. ${ }^{21}$ In such hypertensives blood pressure reduction is not associated with any significant decrease in arterial stiffness. ${ }^{12}$ This could be the reason why some of the subjects of this study have higher PWV even when having normal blood pressure. Failure to reduce PWV with antihypertensive therapy is therefore a significant predictor of cardiovascular morbidity and mortality. ${ }^{22}$

PWV has more of a prognostic significance in addition to diagnosing arterial wall stiffness. ${ }^{23}$ In such individuals, only reduction of blood pressure will not reduce their risk for subsequent cardiovascular events leading to increased morbidity and mortality. Rise of PWV is associated with left ventricular diastolic dysfunction, ${ }^{4}$ left ventricular hypertrophy, ${ }^{12}$ increased myocardial work load, ${ }^{6}$ increased myocardial oxygen demand ${ }^{6}$ but decreased coronary perfusion of the heart. ${ }^{6}$ Interventions targeting modifiable risk factors should be done. A low salt diet, increased intake of antioxidant rich food, and more intake of soy isoflavones ${ }^{24-27}$ and omega-3 fatty acids ${ }^{24,28}$ is most likely to prevent occurrence of hypertension and its complications too. Addition of exercise to one's daily regime will also improve the blood pressure levels and $\mathrm{PWV}, 24,29,30$ as observed by previous studies.

Even if the vascular damage cannot be reversed, further deterioration can be prevented and cardiovascular risk can be brought down significantly.

\section{Limitations of the Study}

Some limitations of this study are as follows:

1. Due to time constraints, a larger sample size could not be taken.

2. Testing of the molecular mechanisms responsible for arterial stiffness is beyond the scope of this study. It should be noted that the mechanisms mentioned earlier are well established by multiple studies.

\section{Conflict of Interest}

None.

\section{Acknowledgments}

We are extremely grateful to WINCARS association for selecting this project under the Prajwalika Scholarship program (PSS). We thank the Department of Physiology at ESIC Medical College, Sanathnagar, Hyderabad for providing their expertise in conducting this study. We sincerely thank the participants of this study.

\section{References}

1 O. Rourke MF, Staessen JA, Vlachopoulos C, Duprez D, Plante GE. Clinical applications of arterial stiffness; definitions and reference values. Am J Hypertens 2002;15(5):426-444

2 Taddei S, Virdis A, Ghiadoni L, et al. Age-related reduction of NO availability and oxidative stress in humans. Hypertension 2001;38(2):274-279

3 Hadi HA, Carr CS, Al Suwaidi J. Endothelial dysfunction: cardiovascular risk factors, therapy, and outcome. Vasc Health Risk Manag 2005;1(3):183-198

4 Seeland U, Brecht A, Nauman AT, et al. Prevalence of arterial stiffness and the risk of myocardial diastolic dysfunction in women. Biosci Rep 2016;36(5):e00400

5 Horváth IG, Németh A, Lenkey Z, et al. Invasive validation of a new oscillometric device (arteriograph) for measuring augmentation index, central blood pressure and aortic pulse wave velocity. J Hypertens 2010;28(10):2068-2075

6 Gurovich AN, Beck DT, Braith RW. Aortic pulse wave analysis is not a surrogate for central arterial Pulse Wave Velocity. Exp Biol Med (Maywood) 2009;234(11):1339-1344

7 Laurent S, Cockcroft J, Van Bortel L, et al; European Network for Non-invasive Investigation of Large Arteries. Expert consensus document on arterial stiffness: methodological issues and clinical applications. Eur Heart J 2006;27(21):2588-2605

8 AlGhatrif $\mathrm{M}$, Strait JB, Morrell $\mathrm{CH}$, et al. Longitudinal trajectories of arterial stiffness and the role of blood pressure: the Baltimore Longitudinal Study of Aging. Hypertension 2013;62(5):934-941

9 Whelton PK, Carey RM, Aronow WS, Casey DE Jr, Collins KJ. Dennison Himmelfarb C. et al, ACC/AHA/AAPA/ABC/ACPM/ AGS/APhA/ASH/ASPC/NMA/PCNA Guideline for the Prevention, Detection, Evaluation and Management of High Blood 
Pressure in Adults-a report of the American College of Cardiology/American Heart Association Task Force on Clinical Practice Guidelines. J Am Coll Cardiol 2018;71(19):•••

10 Chopra HK, Ram CVS, Ram S. Recent guidelines for hypertension. Circ Res 2019;124(7):984-986

11 Cleland JG, Swedberg K, Follath F, et al; Study Group on Diagnosis of the Working Group on Heart Failure of the European Society of Cardiology. The EuroHeart Failure survey programme-a survey on the quality of care among patients with heart failure in Europe. Part 1: patient characteristics and diagnosis. Eur Heart J 2003;24(5):442-463

12 Rabkin SW, Chan SH. Correlation of pulse wave velocity with left ventricular mass in patients with hypertension once blood pressure has been normalized. Heart Int 2012;7(1):e5

13 Kola S, George M, Srinivasamurthy S, et al. Assessment of arterial stiffness index in hypertensive patients in relation to their treatment status attending a tertiary care center in South India. Pharmacol Pharm 2014;5:413-418

14 Harvey A, Montezano AC, Touyz RM. Vascular biology of ageing-implications in hypertension. J Mol Cell Cardiol 2015;83:112-121

15 Wang M, Lakatta EG. Altered regulation of matrix metalloproteinase-2 in aortic remodeling during aging. Hypertension 2002;39(4):865-873

16 Vlassara H, Uribarri J. Advanced glycation end products (AGE) and diabetes: cause, effect, or both? Curr Diab Rep 2014;14(1):453

17 McNulty M, Mahmud A, Feely J. Advanced glycation end-products and arterial stiffness in hypertension. Am J Hypertens 2007;20(3):242-247

18 Corman B, Duriez M, Poitevin P, et al. Aminoguanidine prevents age-related arterial stiffening and cardiac hypertrophy. Proc Natl Acad Sci U S A 1998;95(3):1301-1306

19 Kass DA, Shapiro EP, Kawaguchi M, et al. Improved arterial compliance by a novel advanced glycation end-product crosslink breaker. Circulation 2001;104(13):1464-1470

20 Stewart AD, Millasseau SC, Kearney MT, Ritter JM, Chowienczyk PJ. Effects of inhibition of basal nitric oxide synthesis on carotid-femoral pulse wave velocity and augmentation index in humans. Hypertension 2003;42(5):915-918
21 Benetos A, Adamopoulos C, Bureau JM, et al. Determinants of accelerated progression of arterial stiffness in normotensive subjects and in treated hypertensive subjects over a 6-year period. Circulation 2002;105(10):1202-1207

22 Guerin AP, Blacher J, Pannier B, Marchais SJ, Safar ME, London GM. Impact of aortic stiffness attenuation on survival of patients in end-stage renal failure. Circulation 2001;103(7):987-992

23 Townsend RR, Wimmer NJ, Chirinos JA, et al. Aortic PWV in chronic kidney disease: a CRIC ancillary study. Am J Hypertens 2010;23(3):282-289

24 Wu C-F, Liu P-Y, Wu T-J, Hung Y, Yang SP, Lin GM. Therapeutic modification of arterial stiffness: an update and comprehensive review. World J Cardiol 2015;7(11):742-753

25 Teede HJ, Dalais FS, Kotsopoulos D, Liang YL, Davis S, McGrath BP. Dietary soy has both beneficial and potentially adverse cardiovascular effects: a placebo-controlled study in men and postmenopausal women. J Clin Endocrinol Metab 2001;86(7):3053-3060

26 Teede HJ, McGrath BP, DeSilva L, Cehun M, Fassoulakis A, Nestel PJ. Isoflavones reduce arterial stiffness: a placebo-controlled study in men and postmenopausal women. Arterioscler Thromb Vasc Biol 2003;23(6):1066-1071

27 Nestel P, Fujii A, Zhang L. An isoflavone metabolite reduces arterial stiffness and blood pressure in overweight men and postmenopausal women. Atherosclerosis 2007;192(1):184-189

28 Pase MP, Grima NA, Sarris J. The effects of dietary and nutrient interventions on arterial stiffness: a systematic review. Am J Clin Nutr 2011;93(2):446-454

29 Nocon M, Hiemann T, Müller-Riemenschneider F, Thalau F, Roll S, Willich SN. Association of physical activity with all-cause and cardiovascular mortality: a systematic review and metaanalysis. Eur J Cardiovasc Prev Rehabil 2008;15(3):239-246

30 Mora S, Cook N, Buring JE, Ridker PM, Lee IM. Physical activity and reduced risk of cardiovascular events: potential mediating mechanisms. Circulation 2007;116(19):2110-2118 